\title{
Liquid deposition through evaporation
}

\author{
Asher P. Mouat, ${ }^{*}$ Clay E. Wood, Justin E. Pye, and Justin C. Burton (두 \\ Department of Physics, Emory University, Atlanta, Georgia 30322, USA
}

(Received 30 January 2019; published 24 October 2019)

\begin{abstract}
This paper is associated with a poster winner of a 2018 APS/DFD Gallery of Fluid Motion Award for work presented at the DFD Gallery of Fluid Motion. The original poster is available online at the Gallery of Fluid Motion, https://doi.org/10.1103/APS.DFD.2018. GFM.P0056
\end{abstract}

DOI: 10.1103/PhysRevFluids.4.100512

The deposition of one substance through the evaporation of its solvent has been studied mostly in the context of colloidal particles where coffee-ring structures form due to an evaporation-induced flow towards the contact line [1]. Here we explore a related phenomena: the deposition of a nonvolatile solute liquid due to evaporation of the solvent. During spreading, these binary mixtures are subject to spatially and temporally heterogenous forces due to concentration gradients. The evaporation flux is strongest at the contact line [1], and thus the concentration of the solute liquid is highest in this region. This gradient can lead to Marangoni stresses from the differing liquid surface tensions and a rapidly changing affinity for wetting the substrate $[2,3]$.

Figure 1 shows a partial image of a spreading drop composed of isopropanol (solvent) and ethylene glycol (solute) on a silicon wafer. The isopropanol evaporates much more rapidly because the ethylene glycol has a much lower vapor pressure. The surface tension of isopropanol, $21.5 \mathrm{mN} / \mathrm{m}$, is considerably lower than that of ethylene glycol, $48 \mathrm{mN} / \mathrm{m}$. Thus, the ethylene glycol concentrates at the contact line and "pulls" on the interface due to its higher surface tension, resulting

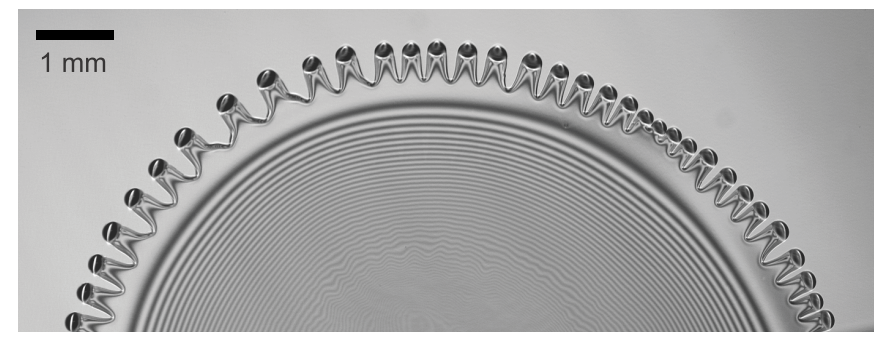

FIG. 1. Partial image of a 1- $\mu 1$ isopropanol drop with 10 vol\% ethylene glycol spreading on a silicon wafer. The fingerlike structures formed at the contact line result from an interplay between evaporation-induced Marangoni stresses and surface wetting. The light and dark regions are interference fringes in the thin liquid film from the monochromatic light used for imaging (the wavelength is equal to $632 \mathrm{~nm}$ ).

*apmouat@emory.edu

Published by the American Physical Society under the terms of the Creative Commons Attribution 4.0 International license. Further distribution of this work must maintain attribution to the author(s) and the published article's title, journal citation, and DOI. 


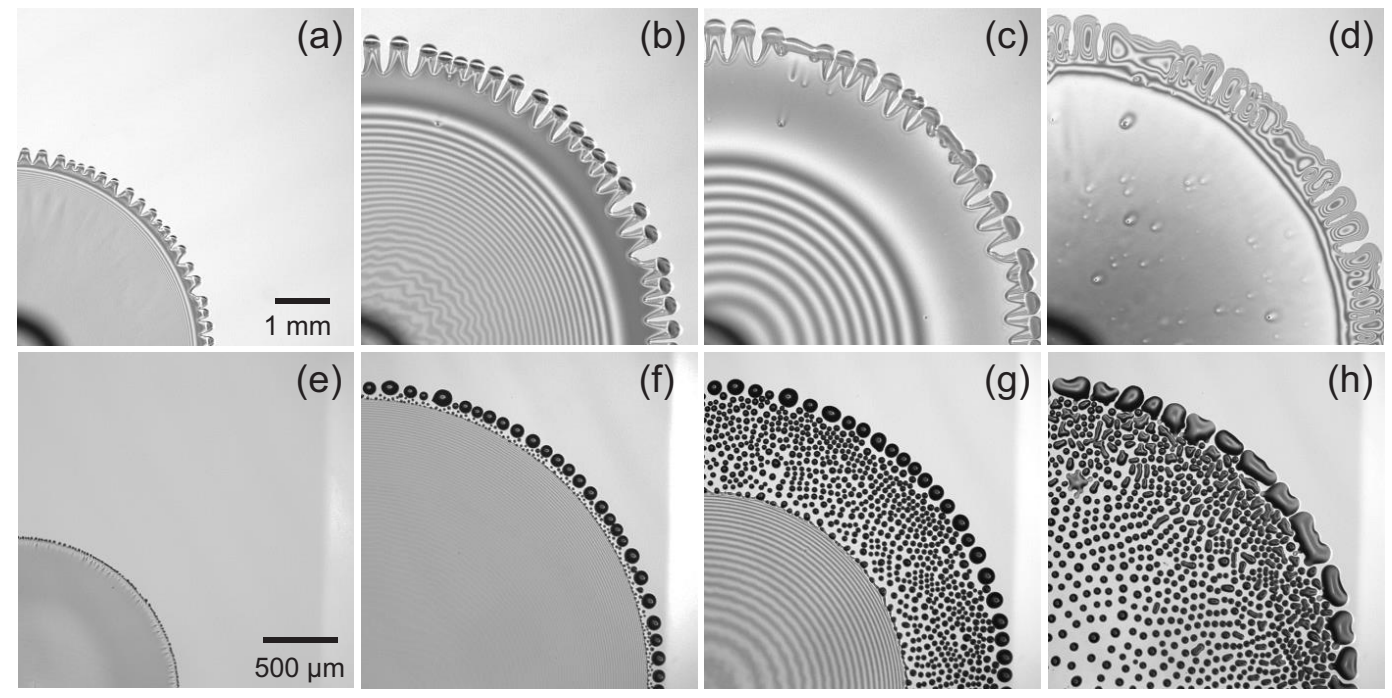

FIG. 2. (a)-(d) Partial images of a 1- $\mu 1$ isopropanol droplet with 10 vol\% ethylene glycol spreading on a silicon surface. (e)-(h) Images of a 1- $\mu$ l isopropanol droplet with 10 vol\% dodecane spreading on a silicon surface.

in the appearance of fingers that spread in advance, connected to the bulk liquid through a very thin film. The grayscale intensity illustrates that the film is very uniform in thickness, since the vertical difference in height between a black and white interference fringe is $113 \mathrm{~nm}$, which is $1 / 4$ of the wavelength of the illuminating monochromatic light inside the liquid with index of refraction approximately equal to 1.4 .

Figures 2(a)-2(d) show partial images during the spreading of an ethylene glycol-isopropanol droplet on a silicon surface, illustrating the full evolution of the fingers, and a thin film of ethylene glycol that remains after the isopropanol has evaporated. In contrast, when dodecane $(25 \mathrm{mN} / \mathrm{m})$ is used as the solute at the same concentration [Figs. 2(e)-2(h)], we instead observe pearls at the contact line since Marangoni forces are no longer dominant. Although pure dodecane wets the silicon surface, in the presence of isopropanol and its saturated vapor, dodecane will retain a finite contact angle until any thin surface film evaporates and diffuses away.

Even with strong Marangoni forces, a mixture of water $(72 \mathrm{mN} / \mathrm{m})$ and isopropanol can display fingers or pearls depending on the surface affinity for each liquid, which we tune by oxygen plasma treatment of the silicon wafer. In Fig. 3(a), the final result is a submicron film of water, which eventually evaporates, whereas the pearls shown in Fig. 3(e) are deposited on the surface. The only difference in the behavior is the amount of time elapsed after oxygen plasma treatment, so
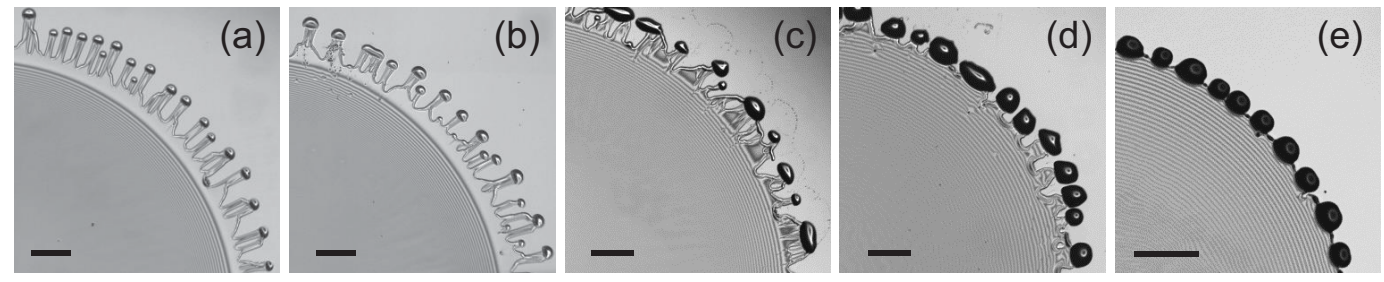

FIG. 3. (a)-(e) Partial images of a 1- $\mu$ l isopropanol drop with 25 vol\% water spreading on an oxygenplasma-treated silicon surface. The waiting time after treatment and before deposition was (a) $0 \mathrm{~h}$, (b) $1 \mathrm{~h}$, (c) $48 \mathrm{~h}$, (d) $96 \mathrm{~h}$, and (e) $144 \mathrm{~h}$. The scale bar in all images is $1 \mathrm{~mm}$. 
the contact angle of the water varies from $10^{\circ}$ to $45^{\circ}$. This sharp transition in behavior, from thin film to droplet deposition, is not fully understood. Nevertheless, the ability to tune between the different behaviors may allow for better control over desired patterns in liquid deposition through evaporation.

[1] R. D. Deegan, O. Bakajin, T. F. Dupont, G. Huber, S. R. Nagel, and T. A. Witten, Capillary flow as the cause of ring stains from dried liquid drops, Nature (London) 389, 827 (1997).

[2] Y. Gotkis, I. Ivanov, N. Murisic, and L. Kondic, Dynamic Structure Formation at the Fronts of Volatile Liquid Drops, Phys. Rev. Lett. 97, 186101 (2006).

[3] L. Keiser, H. Bense, P. Colinet, J. Bico, and E. Reyssat, Marangoni Bursting: Evaporation-Induced Emulsification of Binary Mixtures on a Liquid Layer, Phys. Rev. Lett. 118, 074504 (2017). 